\title{
Mujeres al mando: Cuotas de género y representación descriptiva de las mujeres en América Latina
}

\author{
Female Leadership: Gender Quotas and Women's \\ Descriptive Representation in Latin America
}

\begin{abstract}
RESUMEN
Este estudio aspira a dar luz sobre el impacto diferenciado de las cuotas de género en la representación política de las mujeres latinoamericanas. Para ello, se examina la incidencia del marco electoral sobre la eficacia de estas medidas de acción afirmativa. A partir de indicios que sugieren que el diseño de las leyes de cuotas y la estructura de la papeleta de votación son factores determinantes para potenciar la capacidad de cuotas de género de aumentar la representación descriptiva de las mujeres en el tiempo, se analiza el contexto institucional de trece países en América Latina, a lo largo de sesenta y seis periodos legislativos entre 1991 y 2020. Los países seleccionados son Argentina, Bolivia, Brasil, Chile, Colombia, Costa Rica, Ecuador, El Salvador, Honduras, México, Nicaragua, Perú y Uruguay. El análisis indica que la fortaleza del contenido de las cuotas es un factor particularmente relevante para entender como este mecanismo incide sobre la variación del número de mujeres en el tiempo, independientemente del tipo de lista, mientras que un mayor protagonismo del electorado a través del voto preferencial no perjudica necesariamente el avance hacia la paridad en política.
\end{abstract}

Palabras clave: América Latina, representación descriptiva de las mujeres, cuotas de género.

\section{Abstract}

This study aims at examining the way in which electoral rules affect the impact of gender quotas on female representation in Latin American parliaments. Considering evidence that suggests that a specific legal corpus of the quotas and non-preferential electoral systems are necessary conditions for the effectiveness of gender quotas, the institutional context in thirteen Latin American countries has been examined over a period of time that includes sixty-six legislatures, from 1991 to 2020. The countries that have been selected are Argentina, Bolivia, Brasil, Chile, Colombia, Costa Rica, Ecuador, El Salvador, Honduras, México, Nicaragua, Perú y Uruguay. The results show that the strength of the gender quota design is particularly relevant for understanding how this mechanism affect the variation of the numbers of women over time, no matter the type of orientation of the electoral system, and that voters' greater ability to influence ballot structures through preferential voting does not necessarily represent an obstacle to gender parity.

Keywords: Latin America, women's descriptive representation, gender quotas.

\section{SUMARIO}

1.- Introducción. 2.- Las cuotas de género y el concepto de representación política. 3.- El contenido del diseño legislativo de las cuotas. 4.- La estructura de la papeleta electoral. 5.- La variación de la representación descriptiva de las mujeres latinoamericanas 1991-2020. El análisis. 6.- Conclusiones. - Referencias bibliográficas.

\section{1.- Introducción}


Hoy en día América Latina destaca como la segunda región en el mundo con el porcentaje más alto de mujeres en parlamento, detrás de Escandinavia (Ballivián, 2016: 116-17). Mientras los países escandinavos fueron teatro de las primeras adopciones voluntarias de medidas de acción afirmativas por partes de unos partidos en los años 70, en 1991 Argentina fue el primer país en el mundo a introducir una ley estatal de cuotas de género, que se llamó Ley de Cupos, que obligaba a todos los partidos a incluir un determinado porcentaje de mujeres en las listas de candidatos a las elecciones nacionales. Entre 1996 y 1997, un gran número de países latinoamericanos siguió el ejemplo de Argentina: en línea con las recomendaciones de la Convención para la Eliminación de todas las formas de Discriminación contra las Mujeres en 1979, la Conferencia Mundial de Nairobi en 1985, y especialmente la Cuarta Conferencia Mundial de las Mujeres celebrada en Beijing en 1995, se introdujeron ampliamente las llamadas políticas de acción afirmativa, herramientas institucionales de diferente naturaleza construidas para la reparación por la exclusión sistemática de las mujeres de los espacios de poder a lo largo de la historia hasta nuestros días (Jones et al., 2012: 332-3; Peschard, 2003: 20-21; Tula, 2016: 135-6; Dahlerup y Freidenvall, 2011: 14).

En el panorama latinoamericano la gran mayoría de los países decidió adoptar leyes de cuotas de género, sin embargo, el reto sigue vigente: después de más de veinte años, las mujeres siguen siendo infrarrepresentadas en la arena política aún en la región más a la vanguardia en la aplicación de medidas de acción afirmativa (IDEA International, 2020). De allí, la urgencia de la Agenda 2030 (CEPAL, 2017b; 2017c) en exhortar a todos los estados de «Asegurar la participación plena y efectiva de las mujeres y la igualdad de oportunidades de liderazgo a todos los niveles decisorios en la vida política, económica y pública.» (CEPAL, 2017a: 23).

Además, existen diferencias marcadas en torno a la representación femenina a nivel parlamentario entre los países latinoamericanos (IDEA International, 2020). Según Dahlerup y Freidenvall (2011: 41), si el sistema de cuotas no es compaginable con las reglas electorales vigentes resultaría una medida puramente simbólica. El destino de las cuotas de género parece estrechamente vinculado con el marco institucional en que se desarrolla la medida, y otros factores sistémicos que identifica CEPAL en 2005, cuando afirma que:

Para que la ley de cuotas tenga el efecto esperado es necesario que cumpla con las siguientes condiciones: i) que se adecue al sistema electoral y que esté consagrada en la ley correspondiente; ii) que esté reglamentada adecuadamente; iii) que el organismo electoral vele activamente por su cumplimiento (Zapata, 2007: 42).

Asimismo, los sistemas electorales en América Latina no son compromisos institucionales inamovibles. Desde 1978 se ha observado un proceso de cambio hacia una participación más amplia de la ciudadanía a través de sistemas de voto más personalizados, y una reducción del poder de los partidos políticos (Freidenberg y Došek, 2016: 6-28, 45-6; Miranda Cuesta, 2016: 228; Bareiro et al., 2004: 13-15). No existe certeza de que esta ola de reformas en el contexto electoral a favor de una 
mayor influencia de la ciudadanía sobre los cargos electivos amplifique el impacto de las cuotas sobre la representación descriptiva de las mujeres latinoamericanas, ya que en múltiples estudios se ha argumentado que la inclusión de las mujeres en los espacios políticos se ve a menudo mermada por la tendencia del electorado a votar por un candidato hombre en lugar de una mujer (Fréchette et al. 2008; Jones, 2008: 2; Schmindt-Bayer, 2009; Lauserud y Taphorn, 2007; Autor 1, 2016).

En el esfuerzo de contribuir a descifrar la relación entre la eficacia de las cuotas de género en América Latina y los marcos electorales en que actúan, el presente estudio se propone examinar la variación en el tiempo del número de mujeres en las cámaras bajas de los trece países en la región en que se establecieron leyes de cuotas a nivel nacional. El análisis transnacional comparado incluye sesenta y seis ciclos legislativos desde la introducción de las cuotas hasta el día de hoy, y examina el posible condicionamiento que ejercen elementos institucionales de relevancia como la estructura de la papeleta de votación y el contenido del corpus normativo de las cuotas, sobre la eficacia de estas medidas de acción afirmativa. Los países examinados son Argentina, Bolivia, Brasil, Chile, Colombia, Costa Rica, Ecuador, El Salvador, Honduras, México, Nicaragua, Perú y Uruguay.

En primer lugar, se analiza la relación entre el contenido del corpus normativo de las cuotas y el impacto de estas medidas en la representación descriptiva de las mujeres, para determinar si factores como el porcentaje de representación mínima prefijado, el mandato de posición y la fortaleza de los mecanismos sancionadores, condicionan de manera relevante la evolución del número de diputadas en el tiempo (Jones, 2009; Schwindt-Bayer, 2009; Jones et al., 2012). En este sentido, se plantea como primera hipótesis que, el contenido del diseño legislativo de las cuotas y tres de sus componentes (el porcentaje de representación mínima, el mandato de posición y el tipo de mecanismos sancionadores) son los principales factores de influencia a la hora de examinar la variación de mujeres en cargos electivos en el tiempo, independientemente del tipo de lista.

En segundo lugar, se examina la relación entre la estructura de la papeleta electoral y la eficacia de las cuotas de género, para verificar si los sistemas basados en el voto preferencial afectan o promueven el acceso de las mujeres en el legislativo (Dalton et al., 2011; Karvonen 2004). Con respecto a este punto, la hipótesis que se maneja es que, bajo cuotas de género, independientemente del contenido de esa normativa, la orientación no preferencial o partidaria del sistema electoral incide en mayor medida sobre la representación descriptiva femenina que la orientación preferencial. También, se analiza la interrelación de los dos elementos institucionales examinados y sus incidencias sobre la eficacia de las cuotas de género en términos de variación del número de mujeres parlamentarias latinoamericanas en el tiempo.

En línea con lo dicho, los resultados del análisis apuntan a que las características del diseño de las cuotas que se han evaluado contribuyen a una mayor presencia de las mujeres en el legislativo, en concordancia con la primera hipótesis. Asimismo, los resultados sugieren que los sistemas electorales latinoamericanos donde la población votante tiene la posibilidad de señalar su/s preferencia/s a nivel de 
candidatura, no son un obstáculo a los avances de la representación descriptiva de las mujeres.

\section{2.- Las cuotas de género y el concepto de representación política}

Existe una compleja relación entre representación y género, olvidada por los padres de la ciencia política, que puede ser rescatada a partir de los estudios de Pitkin (1964; 1967). Esta autora, desvinculandose de la visión clásica hobbesiana, enfocada en una autoridad absoluta ejercida sobre un conjunto de representados que le han conferido previamente el poder, aborda este concepto desde un enfoque de rendición de cuenta, y construye un sistema complejo compuesto por cuatro niveles: el formal, descriptivo, sustantivo y el simbólico. El nivel de la representación formal describe el contexto institucional en que los representantes operan, y se puede analizar a partir de las reglas para la selección o remoción de los mismos legisladores (Pitkin, 1967: 1-14; Schwindt-Bayer, 2010: 8; Celis et al., 2008: 3-4). El nivel descriptivo hace referencia a la correspondencia entre la composición interna de las instituciones y la sociedad: se puede hablar tanto de equivalencia territorial o geográfica (Marsh y Wessels, 1997, en Schwindt-Bayer 2010: 8) como de correspondencia en función de características sociales como identidad étnica, de clase o de género (Norris y Franklin, 1997, en Schwindt-Bayer, 2010: 8). La representación sustantiva refleja la capacidad de los legisladores de actuar de manera adecuada según las preocupaciones y demandas de sus electores (Pitkin, 1967: 123-56; Celis et al., 2008: 1-4, 6-8; Wängnerud, 2009: 52; Schwindt- Bayer, 2010: 6, 11-12). El último nivel representa los símbolos que la relación entre representantes y personas representadas puede generar y los sentimientos y actitudes hacia las instituciones que estos símbolos evocan en la ciudadanía (Pitkin, 1967: 101-122; Celis et al., 2008: 3-4; Schwindt-Bayer, 2010: 27-28).

Según Pitkin (1967: 1-14), la representación sustantiva, es decir la manera en que actúan las personalidades representantes, es la dimensión más importante, ya que afecta directamente la capacidad de rendición de cuenta de estos individuos frente a la ciudadanía. Sin embargo, esta dimensión no se puede entender en aislamiento con respecto a niveles como el formal y el descriptivo (Pitkin, 1967; Schwindt-Bayer, 2010: 6-7; Celis et al., 2008: 1, 6-9; Dahlerup y Freidenvall, 2010).

La estrecha relación entre niveles descriptivo y sustantivo es particularmente relevante para el desarrollo del presente estudio. Lejos de los planteamientos esencialistas sobre las inclinaciones innatas de las mujeres que la predispondrían a renovar las prácticas políticas a favor de la cercanía y la empatía, esta investigación toma como referencia posturas interseccionales cercanas a Young (2002). Estas establecen que la participación política femenina es deseable y urgente, ya que las mujeres comparten todas un elemento significativo para la representación: una «perspectiva social» o ubicación social femenina que recoge experiencias y vivencias semejantes debido a las dinámicas de desigualdad que se originan, y al mismo tiempo, están reforzadas por la estructura patriarcal, en la cual los hombres gozan de acceso y control privilegiados a poder y recursos, mientras las mujeres carecen 
de ellos. Por esto, aunque los intereses y las opiniones del colectivo mujeres sean tan heterogéneas que puedan resultar conflictivas, es la «perspectiva social» basada en una memoria social compartida de discriminación y violencia lo que configura una relación preferencial entre mujeres legisladoras y electoras, y que determina la relación estrecha entre representación descriptiva y sustantiva (Young, 2002: 13348; Celis et al., 2008: 6-8; Mansbridge, 2005; Bareiro et al., 2004).

En este sentido, la perspectiva teórica empleada cuestiona los enfoques que describen las mujeres como un grupo homogéneo y estático, tanto desde los planteamientos esencialistas como desde la teoría de la Política de la Presencia de Phillips (1998). Destaca Young (2002: 133-48; Celis et al., 2008: 6-7) que los sujetos políticos que ejercen la representación sustantiva femenina serán con mayor probabilidad mujeres, sin que esto signifique que ellas deban serlo en el sentido biológico. De esta manera, se problematiza la categoría de mujer y se subraya la diferencia entre sexo y género, el primero referido a los atributos sexuales de un cuerpo, y el segundo que indica el conjunto de normas y valores que, en un determinado tiempo y espacio, el orden social asocia a aquellos caracteres sexuales (Celis et al., 2008: 7; Mansbridge, 1999: 637-9; Bacchi, 1996: ix-xv, 9-13). Asimismo, se evidencia la necesidad de prestar particular atención a la interseccionalidad de dimensiones críticas como la clase social, identidad étnica, orientaciones sexuales, entre otras. Este aspecto, por un lado, complejiza las relaciones de poderes entre categorías sociales y, por el otro, desmiente la noción de una supuesta identidad femenina homogénea (Bacchi, 1996: ix-xv; Celis et al., 2008: 6-8; Rai, 2008: 75-7; Phillips, 1995: 52-6). Esta última, revela Mansbridge (2005: 634), ocultaría el punto de vista privilegiado, con su vivencias y demandas, de mujeres blancas, de clase media y con alta formación académica, excluyendo otros tipos de identidades.

Desde los estudios de Mansbridge (1999) y Saward (2006 citado por Celis et al., 2008: 3-6) llegan importantes contribuciones adicionales a esta postura teórica: la representación no funciona de la manera unidireccional descrita por Pitkin, en la cual la comunidad representada tiene demandas que eleva desde las bases hasta las instituciones políticas. Al contrario, los actores políticos son sujetos creativos que tienen una cierta influencia sobre la construcción de intereses y demandas en las bases. A partir de esta premisa, Mansbridge (1999: 635-6) señala que las representantes disfrutan de un bagaje de conocimientos que, basándose en la perspectiva social que comparten con las mujeres votantes, les garantiza una «ventaja comunicativa e informacional» a la hora de tomar decisiones y hacerse portavoz de demandas cercanas y significativas para el electorado femenino. Por consecuencia, una mayor representación descriptiva corresponde a una más elevada representación sustantiva porque cuantas más mujeres en el parlamento, más potencial informativo relevante será puesto en común, creando una «sinergia deliberativa» que podría ser capaz de enfrentar la estructura patriarcal predominante en la arena política y en la sociedad, y generar contextos institucionales democráticos de calidad (635-6).

Para contrastar estas resistencias estructurales y facilitar así el acceso "por vía rápida» de las mujeres en las instituciones públicas, ya se idearon diferentes me- 
canismos de acción afirmativa, entre los cuales destacan las cuotas de género que se han afirmado internacionalmente como una de las principales estrategias para fortalecer la representación política de las mujeres, tanto que hoy en día son ciento treinta los países donde de alguna forma hay cuotas de género vigentes (Lauserud y Taphorn, 2007: 4;IDEA et al., 2020b). En América Latina y el Caribe son veinte los países que han implementado estas medidas: en Haití han sido introducidas cuotas o políticas de asientos reservados; en Guatemala y Venezuela han sido implementadas las cuotas voluntarias; y los restantes diecisiete países han adoptado cuotas legislativas (Jones et al., 2012: 332-3; IDEA et al., 2020a). La primera tipología de cuotas está implementada más a menudo en Asia, Medio Oriente y África, y determina un número de escaños en el parlamento que está garantizado para las mujeres (Dahlerup y Freidenvall, 2010: 416, 2011: 22-23; Archenti y Tula 2007: 187). $\mathrm{Al}$ contrario, las cuotas voluntarias y las cuotas legislativas o legales actúan sobre las listas de candidaturas para las elecciones, ya que exigen que se presente un porcentaje mínimo de mujeres para los cargos electivos. Mientras las primeras son medidas introducidas a discreción del partido, las segundas son normas constitucionales o son parte de reformas de leyes electorales a nivel nacional o federal y, por ello, su cumplimiento es obligatorio (Krook, 2006: 304; Dahlerup y Freidenvall 2011: 21-23).

A pesar de las diferencias, todas estas medidas de acción afirmativa tienen el mérito de ser instrumentos temporales de reparación que promueven la inclusión de un sector mayoritario de la población mundial que, sin embargo, no ha sido representado adecuadamente en política a lo largo de la historia, a causa de un sistema de violencia y discriminación que ha afectado a las mujeres a escala global, no solo limitando sus derechos, sino también marginalizándolas de los espacios de poder (Archenti y Tula, 2013; CEPAL, 2016: 138-42; Bareiro et al., 2004: 25; Tula, 2016). Como resultado, el acceso femenino a los diferentes ámbitos públicos a nivel global ha sido, y sigue siendo, desigual, ya que «cuando se tienen distintas condiciones de partida, no se genera una competencia en igualdad de oportunidades» (CEPAL, 2016: 139). En regímenes donde la democracia es un concepto amplio y profundo que incorpora la participación inclusiva y plural del conjunto de la ciudadanía y no se limita al mero ejercicio electoral cada cuatro o cinco años, esta desigualdad estructural y sistemática no puede ser aceptada (Mouffle, 1991 y Waylen, 1994, en Bareiro et al., 2004: 13-22; Dahl, 1971 en Disch, 2011: 100, 108). En línea con acuerdos internacionales como el Consenso de Quito de 2007, y con las notables aportaciones de teóricas feministas como Young (2002) y Mansbridge (2005), el estudio se sustenta en la certeza que la presencia de las mujeres en los órganos de toma de decisión es un elemento determinante para la calidad de la representación política en democracia, y que mecanismos de acción afirmativa como las cuotas de género son instrumentos valiosos para conseguir este objetivo (Archenti y Tula, 2013: 6-7; CEPAL, 2016: 139).

3.- El contenido del diseño legislativo de las cuotas 
La literatura ofrece evidencias convincentes de que los regímenes de cuotas de género deben incorporar requisitos adecuados para incidir de manera significativa, siendo el valor porcentual de representación que las cuotas establecen uno de los factores que primero destaca (Schwindt-Bayer, 2010: 47; Archenti y Tula, 2007; Larserud y Taphorn, 2007; Jones, 2009; Schwindt-Bayer, 2009; Htun y Jones, 2002; Archenti y Tula, 2013; Jones et al., 2012, entre otros).

Asimismo, en el análisis comparado de Jones (2009) sobre veintinueve cámaras altas y bajas en América Latina, se distingue entre cuotas bien dibujadas y cuotas laxas, identificando como factor determinante la presencia de mandato de posición. Esta medida ejerce un condicionamiento notable sobre la probabilidad de que los candidatos ocupen puestos privilegiados en la papeleta y que, en consecuencia, tengan mayores posibilidades de ser elegidos, ya que indica en que orden los postulantes de uno u otro sexo estarán ubicado a lo largo de la lista. En Argentina, por ejemplo, apenas dos años después de la Ley de Cupo Femenino de 1991, se aprobó el decreto N. ${ }^{\circ} 379$ de 1993 para especificar que en las listas plurinominales un candidato cada tres debía ser mujer, y en el caso de los distritos binominales, uno solo de los dos presentados podía ser varón (IDEA et al., 2020c). En Honduras, la Ley de Igualdad de Oportunidades para la Mujer de 2000 precisa en el Art. 81 que las mujeres deben ocupar lugares con expectativas en la papeleta de votación, basándose en número de asientos que cada partido haya ganado en las últimas tres elecciones (Archenti, 2011: 3). Estas medidas han sido pensadas para prevenir prácticas evasivas de los partidos políticos que, aunque cumpliendo con lo preestablecido en las cuotas de género, no respaldan con sinceridad el espíritu de esas políticas y se limitan a colocar el número requerido de candidatas al final de la papeleta, perjudicando su probabilidad de ser elegidas (Archenti y Tula, 2013: 2-6; Tula, 2016).

En el estudio transnacional de Htun y Jones (2002) se argumenta que las cuotas deben incluir normas sancionadoras estrictas para ser efectivas. Lo mismo sugiere Schwindt-Bayer (2010: 51-55) cuando presenta un índice que mide el grado de rigor de los instrumentos de sanción, diferenciando entre mecanismos no presentes, mecanismos débiles y mecanismos fuertes. El máximo rigor se identifica con la presencia de instituciones monitoras independientes que tengan la autoridad de invalidar las listas que no cumplan con los requisitos de las cuotas y de negar la inscripción a todas las candidaturas en su conjunto.

En línea con la literatura analizada, en este estudio se examina cómo los tres componentes fundamentales de las cuotas, es decir, el porcentaje de representación mínima, la presencia de mandatos de posición y de mecanismos sancionadores por no conformidad, inciden en la medida en que el instrumento legal influye en la representación femenina. A partir de estas dimensiones, se ha construido un índice de elaboración propia sobre la Fuerza de las Cuotas de Género que permite diferenciar entre cuotas de género laxas (valor: 0) o fuertes (valor: 1), como se evidencia en la Tabla 1, construida teniendo en cuenta las decretos, reglamentos y leyes electorales 
de cada país ${ }^{2}$.

Tabla 1. Cuotas de género

\begin{tabular}{|c|c|c|c|c|c|}
\hline PAÍS & $\begin{array}{c}\text { AÑO DE } \\
\text { INTRODUC- } \\
\text { CIÓN O } \\
\text { REFORMA }\end{array}$ & $\begin{array}{l}\text { PORCENTAJE } \\
\text { MÍNIMO DE } \\
\text { REPRESENTA- } \\
\text { CIÓN }\end{array}$ & $\begin{array}{l}\text { MANDATO DE } \\
\text { POSICIÓN }\end{array}$ & $\begin{array}{c}\text { MECANISMOS } \\
\text { SANCIONAD. }\end{array}$ & $\begin{array}{c}\text { CUOTAS } \\
\text { LEGIS- } \\
\text { LATIVAS } \\
\text { LAXAS O } \\
\text { FUERTES }\end{array}$ \\
\hline \multirow[t]{2}{*}{ Argentina } & $1991-2000$ & 30 & Si (1 cada 3$)$ & Fuertes & 1 \\
\hline & 2017 & 50 & L. Cremallera & Fuertes & 1 \\
\hline \multirow[t]{2}{*}{ Bolivia } & 1997 & 30 & Si (1 cada 3 ) & Fuertes & 1 \\
\hline & 2010 & 50 & L. Cremallera & Fuertes & 1 \\
\hline Brasil & $1997-2009$ & 30 & No & Débiles & 0 \\
\hline Chile & 2015 & 40 & No & Fuertes & 1 \\
\hline Colombia & 2011 & $\begin{array}{c}30 \text { (distritos con } 5 \\
\text { o más curules) }\end{array}$ & No & No & 0 \\
\hline \multirow[t]{2}{*}{ Costa Rica } & 1996 & 40 & No & No & 0 \\
\hline & 2009 & 50 & L. cremallera & Fuertes & 1 \\
\hline \multirow[t]{3}{*}{ Ecuador } & 1998 & 20 & No & No & 0 \\
\hline & 2000 & $\begin{array}{r}30(+5 \% \text { en cada } \\
\text { proceso electoral })\end{array}$ & Si (1 cada 3 ) & Fuertes & 1 \\
\hline & 2009 & 50 & L. cremallera & Fuertes & 1 \\
\hline El Salvador & 2013 & 30 & No & Débiles & 0 \\
\hline \multirow[t]{4}{*}{ Honduras } & 2000 & 30 & No & No & 0 \\
\hline & 2004 & 30 & No & Débiles & 0 \\
\hline & 2012 & 40 & No & Débiles & 0 \\
\hline & 2016 & 50 & $\begin{array}{c}\text { Si (cremal. } \\
\left.\text { desde } 3^{\text {a }}, 4,5\right)\end{array}$ & Débiles & 1 \\
\hline México & 1996 & 30 & No & No & 0 \\
\hline
\end{tabular}

2 Ley N $\mathrm{N}^{\circ}$ 24012, 1991; Decreto No 379, 1993; Decreto No 1246, 2000; Ley No 27412, 2017

(Argentina). Ley No 1779, 1997; Ley No 026, 2010 (Bolivia). Ley Nº 9.504, 1997; Ley 12.034, 2009 (Brasil). Ley 20840, 2015 (Chile). Ley N ${ }^{\circ}$ 1475, 2011 (Colombia). Ley No 7.653, 1996; Ley No 8.765, 2009 (Costa Rica). Constitución Política de la República de Ecuador, 1998; Ley Orgánica Electoral s/n, 2009; El Reglamento General a la Ley de Elecciones, 2000 (Ecuador). Ley No 307 (El Salvador). Ley de Igualdad de Oportunidades para la Mujer, 2000; Ley Electoral y de las Organizaciones Políticas, Decreto 44, 2004; Decreto 54, 2012; Reglamento 003, 2016 (Honduras). Código Federal de Instituciones y Procedimientos Electorales COFIPE, 1996; Reforma al Código Federal de Instituciones y Procedimientos Electorales, 2002; Código Federal Electoral 2008; Ley General de Instituciones y Procedimientos Electorales, 2014 (México). Ley No 26.859, 1997; Ley Nº 648, 2008; Decreto 29, 2010; Ley No 790, 2012 (Nicaragua). Ley $\mathrm{N}^{\mathrm{o}}$ 26859, 1997;

Ley No 27387, 2000; Ley Nº 30996, 2019 (Perú). Ley Nº 18476, 2009 (Uruguay). 


\begin{tabular}{|c|c|c|c|c|c|}
\hline & 2002 & 30 & Si (cada 3) & Débiles & 0 \\
\hline & 2008 & 40 & Si (2 cada 5) & Débiles & 1 \\
\hline & $2014-2017$ & 50 & L. Cremallera & Débiles & 1 \\
\hline \multirow[t]{2}{*}{ Nicaragua } & 2008- 2010 & 50 & No & No & 0 \\
\hline & 2012 & 50 & L. Cremallera & No & 1 \\
\hline \multirow[t]{2}{*}{ Panamá } & 1997 & $\begin{array}{c}30 \text { (solo prima- } \\
\text { rias) }\end{array}$ & No & No & - \\
\hline & 2012 & $\begin{array}{c}50 \text { (solo prima- } \\
\text { rias) }\end{array}$ & No & No & - \\
\hline Paraguay & 1996 & $\begin{array}{c}20 \text { (solo prima- } \\
\text { rias) }\end{array}$ & Si (1 cada 5) & Fuertes & - \\
\hline \multirow[t]{3}{*}{ Perú } & 1997 & 25 & No & Débiles & 0 \\
\hline & 2000 & 30 & No & Débiles & 0 \\
\hline & $\begin{array}{c}2019 \text { (a partir } \\
\text { de } 2021 \text { ) }\end{array}$ & 40 & L. Cremallera & Débiles & 1 \\
\hline \multirow[t]{3}{*}{$\begin{array}{l}\text { República } \\
\text { Domini- } \\
\text { cana }\end{array}$} & 1997 & $\begin{array}{l}25 \text { (nacional., } \\
\text { no por circun- } \\
\text { scripc.) }\end{array}$ & No & No & - \\
\hline & 2000 & $\begin{array}{l}33 \text { (nacional., no } \\
\text { por circun- } \\
\text { scripc.) }\end{array}$ & No & Débiles & - \\
\hline & 2018 & 40 & No & Débiles & - \\
\hline Uruguay & $2009-2017$ & 33 & Si (1 cada 3 ) & Fuertes & 1 \\
\hline
\end{tabular}

Elaboración propia según leyes electorales de cada país, bases de datos de Quotaproyect, Inter- parliamentary Union e IDEA International; Archenti y Tula, 2013.

El primer componente del Índice de la Fuerza de las Cuotas de Género describe el porcentaje de representación mínima que quieren alcanzar las cuotas, asignando el valor 1 a porcentajes hasta el $25 \%, 2$ a valores entre 26 y $39 \%$ y 3 a la paridad (entre 40 y $60 \%$ ). La segunda dimensión muestra el estado del mandato de posición asignándole los valores ordinales 1 , 2 y 3 según el instrumento esté ausente, presente o exija listas cremalleras respectivamente. El tercer componente describe el grado de fuerza de los mecanismos sancionadores (1- que no hay, 2- débiles, 3- fuerte). En su conjunto, el índice registra valores de 4 a 6 para las cuotas laxas, y de 7 a 9 para las cuotas fuertes o bien dibujadas, y finalmente en la Tabla 4 se agrupan los varios escenarios en dos categorías dicotómicas (0- cuotas laxas, 1- cuotas fuertes).

\section{4.- La estructura de la papeleta electoral}


En la literatura un segundo elemento institucional considerado muy significativo a la hora de examinar el impacto de las cuotas de género es la estructura de la papeleta de votación (Jones y Navia, 1999; Archenti y Tula, 2008; Schwindt-Bayer, 2010; Jones, 2009; Schmidt, 2003; Kittilson, 2006; Larserud y Taphorn, 2007; Jones, 2009; Schmidt, 2003; Fréchette et al., 2008; Jones et al., 2012). Muy frecuentes son las comparaciones entre dos categorías contrapuestas: los sistemas de listas cerradas y bloqueadas, por un lado, y las listas abiertas, por el otro. En los primeros, son los partidos quienes determinan el orden de los candidatos en la papeleta, y de esa forma, limitan o favorecen la elección de unos respecto a otros. El electorado, por su parte, tiene derecho a un solo voto que se traduce en su preferencia entre agrupaciones predeterminadas. Al contrario, los sistemas de listas abiertas permiten que quienes voten seleccionen un determinado número de candidaturas, según su orden de preferencia (Archenti y Tula, 2007: 190; Soldevilla, 2005: 220-1; Jones y Navia, 1999; Schmidt, 2003; Larserud y Taphorn, 2007; Pachano, 2004: 7-8).

Cabe subrayar que la frecuente distinción entre listas abiertas y cerradas deja afuera varios sistemas electorales que no encajan en esta contraposición dicotómica. Por ello, el estudio examina la relación entre la variación de participación de las mujeres en el legislativo y la orientación del sistema electoral, basada en un espectro que mide el grado de centralidad de los partidos contrapuesta a la del electorado, desde la perspectiva de Farrell y McAllister (2006). De acuerdo con su Índice de Orientación Preferencial, los sistemas preferenciales, en que el electorado tiene la facultad de dar su preferencia a nivel de candidaturas, están contrapuestos a los sistemas no preferenciales o partidarios, donde la ciudadanía asigna su voto a una lista y su capacidad de influencia es mucho más limitada (Farrell y Scully, 2007: 7-11; Farrell y McAllister, 2006; Dalton et al., 2011). De acuerdo con la Tabla 2, a un extremo del espectro están los sistemas preferenciales puros (Voto Único Transferible, Voto Alternativo, Lista abierta o Panachage), entre los valores 10 y 7.1, y al otro extremo se encuentran los sistemas de voto no preferencial (Lista Cerrada y No bloqueada, Lista Cerrada y Bloqueada), entre 2.9 y 1.4, mientas los valores centrales están asignados a los sistemas mixtos.

Tabla 2. Índice de Orientación Preferencial 


\begin{tabular}{|c|c|c|c|}
\hline Index & Component scores & Description of system & Cases \\
\hline 10 & $4,5,3$ & STV & Ireland \\
\hline 8.6 & $4,5,2$ & AV & Australia \\
\hline 7.1 & $2,4,3$ & Open list, panachage & Denmark, Switzerland \\
\hline 5.7 & $3,3,2$ & SSD, two rounds & France, USA \\
\hline 4.7 & [MM fix A] & MMS with runoff rule & Hungary, Lithuania \\
\hline 4.3 & $1,3,2$ & $\begin{array}{l}\text { SSD-plurality with } \\
\text { party control }\end{array}$ & Canada, UK \\
\hline \multirow[t]{2}{*}{3.6} & [MM fix B] & MMS with plurality rule & \\
\hline & & & $\begin{array}{c}\text { Germany, Japan, Korea, Mexico, New Zealand, } \\
\text { Taiwan, Thailand }\end{array}$ \\
\hline 2.9 & $2,2,1$ & Ordered list & $\begin{array}{c}\text { Belgium, Czech Republic, Netherlands, Poland, } \\
\text { Slovenia, Sweden }\end{array}$ \\
\hline 1.4 & $1,1,1$ & Closed list & $\begin{array}{l}\text { Bulgaria, Iceland, Israel, Norway, Portu- } \\
\text { gal, Romania, Spain }\end{array}$ \\
\hline
\end{tabular}

Fuente: Table 1: Scoring Electoral Systems on the Intra-Party Dimension, in Farrell and McAllister 2006, 7; Dalton et al., 2011, 44.

La literatura ofrece evidencias persuasivas sobre la mayor eficiencia de los sistemas de lista cerrada y bloqueada bajo cuotas de género (Archenti y Tula, 2007: 190; Jones y Navia, 1999; Jones et al., 2012; Jones, 2009; Htun y Jones, 2002; Larserud y Taphorn, 2007). En particular, en presencia de mandato de posición y mecanismos sancionadores apropiados, esta configuración garantiza un nivel mínimo de representación descriptiva femenina que, según los cálculos, variaría entre el 20 y el 50\%, ya que no permite cambiar el orden de los candidatos previamente preestablecidos por el mandato de posición. Al contrario, ningún nivel de representación mínima puede ser asegurado en sistemas de lista abierta, donde solo las preferencias del electorado inciden en el orden de selección (Htun y Jones 2002; Schwindt-Bayer, 2010: 55-60).

Además, varios estudios demuestran que las cúpulas de los partidos pueden actuar como un importante trampolín para las cuotas de género, debido a afinidades ideológicas (Caul, 2001), a presiones internacionales (Krook, 2006; Paxton et al., 2010), o como estrategia para asegurarse un mayor apoyo del electorado (Murray, 2010: 21; Kittilson, 2006; Matland y Studlar, 1996). Los partidos políticos son actores claves para el posible fomento de la representación femenina por el rol de gatekeepers que ejercen: controlan la configuración de las listas electorales, además son espacios donde se crean alianzas y oportunidades de carrera que frenan o am- 
pararan el ascenso de los candidatos (Caul, 2001; Kittilson, 2006; Dahlerup y Freidenvall, 2008: 17-8; Archenti y Tula, 2013: 5). En el desdichado caso de que estas organizaciones violaran el espíritu de las cuotas en su implementación, perjudicarían enormemente su eficacia y contrarrestarían los avances en materia de representación femenina, aunque las condiciones institucionales fueran las más favorables e incorporaran un sistema de lista cerrada y bloqueada además de mandatos de posición y sanciones adecuadas (Matland, 1998: 71, en Archenti y Tula, 2007: 188; Freidenberg y Del Valle, 2017; Archenti y Tula, 2013; Archenti, 2011; Freidenberg et al., 2018).

Por otro lado, cuando el electorado apoya las políticas de acción afirmativa, los sistemas de lista abierta favorecen un mayor impacto de las cuotas en la participación política de las mujeres (Archenti y Tula, 2007: 190-1; McElroy y Marsh, 2009). En el contexto latinoamericano, importantes encuestas como las de InterAmerican Dialogue en 2000 (Peschard, 2003: 20-29), las de World Value Survey entre 2000 y 2004 (World Value Survey, 2020a, 2017b), y las que fueron llevadas a cabo por CEPAL diez años más tardes (Archenti, 2011; Archenti, 2009) demuestran que vastos sectores de la opinión pública apoya la implementación de medidas como las cuotas de género y no considera que los hombres sean mejores líderes. Sin embargo, aún no hay consenso sobre la influencia positiva de las listas abiertas en América Latina (Archenti y Tula, 2007: 190-1).

En línea con las aportaciones de las investigaciones analizadas, este estudio examina en qué manera el grado de orientación preferencial afecta al impacto de las cuotas de género sobre la representación política de las mujeres latinoamericanas. Como detalla la Tabla 3, se asigna el valor 0 a la categoría de los Sistemas preferenciales $(\mathrm{N}=31)$ que recoge las listas abiertas, los panachage, y los sistemas mixtos de Bolivia y México, y el valor 1 a los Sistemas partidarios ( $\mathrm{N}=35)$ que incluye a las listas cerradas y bloqueadas, las listas cerradas y no bloqueadas, y a los sistemas proporcional mixtos.

Tabla 3. Sistemas electorales según la estructura de papeleta electoral

\begin{tabular}{|c|c|c|c|}
\hline País & $\begin{array}{l}\text { Cuotas género } \\
\text { legislativa o } \\
\text { voluntaria }\end{array}$ & Sistema electoral & $\begin{array}{l}\text { Orientación: } \\
\text { preferencial 0, } \\
\text { partidaria } 1\end{array}$ \\
\hline Argentina & $\mathrm{L}$ & RP: lista cerrada y bloqueada (desde 1983). & 1 \\
\hline Bolivia & $\mathrm{L}$ & $\begin{array}{c}\text { RP: lista cerrada y bloqueada (1967-94); mixto (reforma } \\
\text { 1994). }\end{array}$ & $1 ; 0$ \\
\hline Brasil & $\mathrm{L}$ & RP: lista abierta (desde 1986). & 0 \\
\hline Chile & $\mathrm{L}$ & $\begin{array}{l}\text { Binominal: Dos candidatos por lista, voto preferencial } \\
\text { (desde 1980); PR: } 1 \text { voto por un candidato, lista cerrada y } \\
\text { no bloqueada (reforma 2015). }\end{array}$ & 1 \\
\hline
\end{tabular}




\begin{tabular}{|c|c|c|c|}
\hline Colombia & $\mathrm{L}$ & $\begin{array}{l}\text { RP: } 1 \text { voto preferencial (lista cerrada y no } \\
\text { bloqueada) desde 1994; mixto proporcional (reforma } \\
\text { 2003). }\end{array}$ & 1 \\
\hline Costa Rica & $\mathrm{L}$ & RP: lista cerrada y bloqueada (desde 1949). & 1 \\
\hline Ecuador & $\mathrm{L}$ & RP: panachage/voto preferencial (desde 1998). & 0 \\
\hline El Salvador & $\mathrm{L}$ & $\begin{array}{c}\text { RP: lista cerrada y no bloqueada (desde 2011); mixto } \\
\text { proporcional (reforma 2015). }\end{array}$ & 1 \\
\hline Honduras & $\mathrm{L}$ & $\begin{array}{l}\text { RP: lista cerrada y bloqueada (desde 1988), } \\
\text { lista abierta (2004). }\end{array}$ & $1 ; 0$ \\
\hline México & $\mathrm{L}$ & Mixto o segmentado desde 1962. & 0 \\
\hline & & $\begin{array}{c}\text { Nicaragua } \\
\text { L } \\
\text { RP: lista cerrada y bloqueada (desde 1987). } \\
\end{array}$ & \\
\hline Panamá & $\mathrm{L}$ & Mixto (desde 1983). & - \\
\hline Paraguay & $\mathrm{L}$ & RP: lista cerrada y bloqueada (desde 1992) & - \\
\hline Perú & $\mathrm{L}$ & $\begin{array}{c}\text { RP: lista cerrada, no bloqueada, voto preferencial } \\
\text { Doble (desde 1984/93); Doble voto preferencial } \\
\text { Opcional (2000); Lista } \\
\text { cerrada, no bloqueada, voto preferencial y Opcional } \\
\text { (2009). }\end{array}$ & 1 \\
\hline $\begin{array}{l}\text { República } \\
\text { Dominicana }\end{array}$ & $\mathrm{L}$ & $\begin{array}{l}\text { RP: lista cerrada y bloqueada (1994-2010); } \\
\text { voto preferencial (reforma 2010). }\end{array}$ & - \\
\hline Uruguay & $\mathrm{L}$ & $\begin{array}{l}\text { RP: lista cerrada y bloqueada. Ley de lemas } \\
\text { (desde }\end{array}$ & 1 \\
\hline
\end{tabular}

Elaboración propia según leyes electorales del país, bases de datos de Inter-parliamentary Union y Aceproject, Casas -Zamora et al., 2016.

En los sistemas panachage se permite al electorado seleccionar tantos candidatoscomo cargos disponibles haya, independientemente de la lista a que pertenezcan, como en el caso de Ecuador (Pachano, 2004: 7-8; Ballivián, 2016: 1045; Larserud, y Taphorn, 2007: 12-3). En los sistemas electorales mixtos se combinan las características de los sistemas de representación proporcional para las circunscripciones plurinominales, y de los sistemas mayoritarios para los distritos uninominales (Casas-Zamora et al., 2016: 291-331; Alcántara et al., 2008: 21-79, 289319; Alcántara y Tagina, 2013: 289-334). Finalmente, en los sistemas de lista cerrada y no bloqueada, se permite al electorado indicar su preferencia con respecto a un número muy limitado de candidaturas, sin que se permita la movilidad entre agrupaciones, $\mathrm{y}$ en los sistemas proporcionales mixtos se asigna un voto a una lista cerrada y bloqueada, y un segundo, opcional, a un candidato (Casas-Zamora et al., 2016: 291-331; Soldevilla, 2005: 220-1; Archenti y Tula, 2007: 189-91). 


\section{5.- La variación de la representación descriptiva de las mujeres latinoamericanas} 1991- 2020. El análisis.

El análisis se ha llevado a cabo a nivel de periodos legislativos y los resultados incluyen trece países por un total de sesenta y seis legislaturas, desde el año en que se estableció cada reforma de cuotas de género hasta el día de hoy. El aumento o la disminución en el tiempo la representación femenina se determina por la variación positiva o negativa del número de mujeres elegidas como diputadas en un periodo legislativo con respecto al anterior, a lo largo de casi treinta años, desde 1991 a 2020.

En la Tabla 4 se aprecia cómo cada una de las dos variables independientes, primero la fortaleza de las cuotas y luego la orientación de los sistemas electorales, afecta de una determinada manera la variación de representación política de las mujeres latinoamericanas en los trece países analizados. Al examinar la fortaleza del contenido de las cuotas de género, se pueden observar los promedios de la variación porcentual entre treinta y ocho, y entre veintiocho periodos legislativos respectivamente, según las categorías identificadas (4,29\% y 3,35\%), y también los valores de la desviación estándar correspondiente. El primer grupo, de las cuotas fuertes, incluye todas las legislaturas celebradas en Bolivia desde 1997 y en Argentina desde 1993, además de los periodos legislativos de Costa Rica desde 2010, de Chile desde 2017, de Ecuador desde 2002, de Honduras desde 2017, de México desde 2009, de Nicaragua desde 2016, y de Uruguay desde 2014. En el segundo grupo, de las cuotas laxas, se recogen los periodos legislativos de Brasil desde 1998, Colombia desde 2014, de Costa Rica de 1998 a 2010, de Ecuador de 1998 a 2002, de El Salvador desde 2015, de Honduras de 2001 a 2017, de México de 2000 a 2009, de Nicaragua de 2011 a 2016, y de Perú desde 2000.

Cuando consideramos la orientación del sistema electoral, se puede observar los promedios de la variación porcentual del número de mujeres con respecto a treinta y cinco legislaturas celebradas en sistemas electorales partidarios $(3,54 \%)$, y con respecto a treinta y uno periodos legislativos desarrollados en sistemas que promueven el voto preferencial por parte del electorado (4,26\%). Se incluye también los valores de la desviación estándar para cada categoría. El primer grupo, de los sistemas partidarios, consiste en los periodos legislativos celebrados en Argentina, Chile, Colombia, Costa Rica, El Salvador, Honduras de 2001 a 2005, Nicaragua, Perú y Uruguay. El segundo grupo, de los sistemas preferenciales, comprende Bolivia, Brasil, Ecuador, Honduras desde 2005, y México. 
Tabla 4. Variación del porcentaje de mujeres de un periodo legislativo a otro

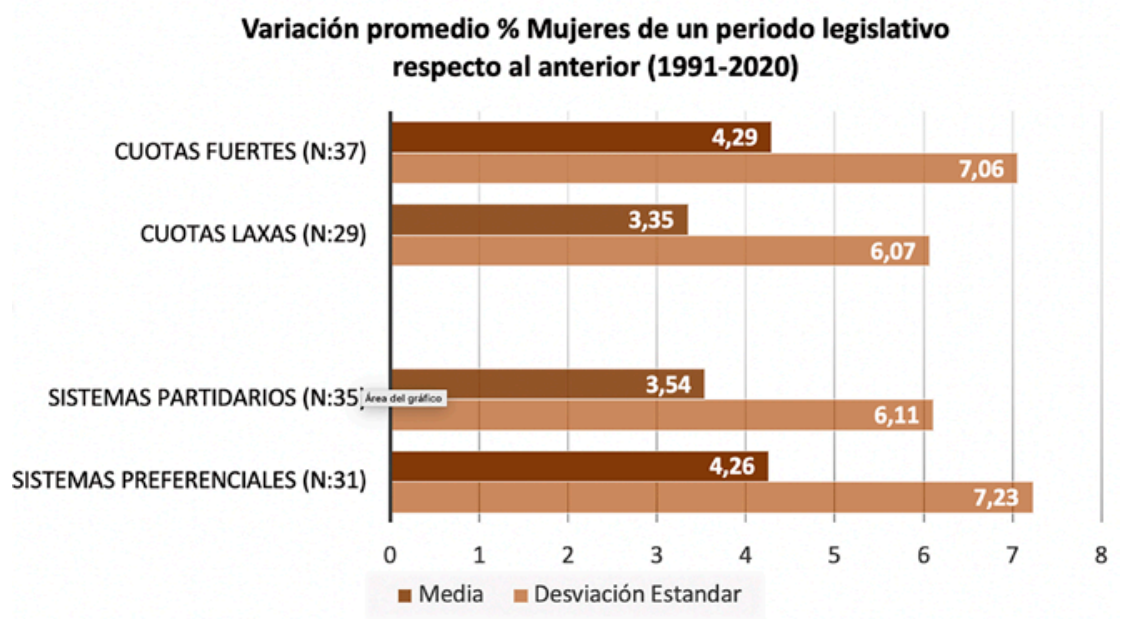

Elaboración propia según leyes electorales del país, bases de datos de Quotaproyect, IDEA International, Inter-parliamentary Union y Aceproject; Casas-Zamora et al., 2016; Archenti y Tula, 2013.

De acuerdo con la tabla, en los países donde se han adoptados leyes de cuotas se observa un significativo aumento promedio del número de legisladoras, elección tras elección. Además, se pone de relieve que el diseño y las características propias de las medidas de cuotas de género son significativas a la hora de examinar el aumento de mujeres en las cámaras: en los escenarios donde se han establecido cuotas de género fuertes, el aumento promedio entre periodos legislativos es más amplio, de acuerdo con la primera hipótesis del estudio. Con respecto a la segunda hipótesis, la Tabla 4 pone en evidencia que, el promedio de la variación en la representación femenina entre periodos legislativos bajo cuotas de género legales resulta más amplio en los sistemas con orientación partidaria que en los preferenciales, con una diferencia de $0,72 \%$. Esta observación sugiere que en los sistemas electorales latinoamericanos donde los partidos tienen menos control sobre la papeleta de votación, las cuotas legislativas inciden en mayor medida en el número de mujeres en el legislativo.

Esta última observación engarza con las posturas discriminatorias y evasivas que se han registrado a lo largo del tiempo dentro de los partidos políticos latinoamericanos (Archenti y Tula, 2008, en Archenti, 2011: 52-54; Archenti y Tula, 2013; Freidenberg y Del Valle Pérez 2017; Freidenberg et al., 2018). De acuerdo con Archenti y Tula (2013: 4-6), se ha demostrado que las cúpulas de los partidos violan las normativas de las cuotas a través de su interpretación minimalista de la ley, puesto que en la mayoría de los casos interpretan los porcentajes de representación mínima de las cuotas como 
techos máximos. A eso se adjunta el «cumplimiento burocrático» de los requisitos, como por ejemplo reiterando el mandato de posición mínimo a lo largo de toda la lista (Archenti y Tula: 4-6). Además, existen partidos políticos que se degradan realizando verdaderas prácticas de acoso político: se registran casos en que a las candidatas se les asignan distritos con escasas posibilidades de éxito o se les fuerza a renunciar a los cargos una vez elegidas. En otros casos, los partidos vulneran la eficacia de las cuotas de género incorporando nombres de mujeres para candidatos hombres, como por ejemplo ocurría en Bolivia antes de la reforma constitucional de 2009 (Archenti, 2011; Archenti y Tula, 2013: 12). Otras prácticas graves de acoso político en la región comprenden intimidaciones, amenazas y agresiones durante y después de las campañas electorales, el trato discriminatorio por parte de los medios de comunicación y la asignación a comisiones secundarias, con bajo presupuesto o sin él (CEPAL, 2016: 137-8; Freidenberg y Del Valle Pérez, 2017).

La siguiente tabla engloba las dos variables independientes, teniendo en cuenta la orientación de los sistemas electorales a la vez que la fortaleza de las cuotas, para dar luz sobre el tipo de contexto electoral en que las cuotas ejercen un impacto mayor en la representación femenina en el legislativo. Para ello, en el análisis se ha construido cuatros modelos o escenarios para explorar las siguientes combinaciones: i) cuotas laxas y orientación preferencial del sistema electoral; ii) cuotas fuertes y orientación preferencial; iii) cuotas laxas y orientación partidaria; iv) cuotas fuertes y orientación partidaria. El escenario I corresponde a catorce ciclos legislativos celebrados en Brasil desde 1998, en Honduras de 2005 a 2017, y México de 1997 a 2009; y el segundo comprende diecisiete legislaturas que incluyen a las de Bolivia (desde 1997), Ecuador (desde 2002), Honduras (desde 2017); y México (desde 2009). El escenario III se compone de quince muestras que corresponden a Colombia (desde 2014), Costa Rica (de 1998 a 2010), El Salvador (desde 2015), Honduras (de 2001 a 2005), Nicaragua (de 2011 a 2016), y Perú (desde 2000), mientras que el último escenario abarca veinte periodos legislativos que comprenden Argentina (desde 1993), Chile (desde 2017), Costa Rica (desde 2010), Nicaragua (desde 2016), y Uruguay (desde 2014).

La Tabla 5 resume los cuatros modelos de análisis y pone de relieve que las condiciones más favorables son presentadas en el escenario II (cuotas fuertes, orientación preferencial débil) que representa una variación porcentual promedio del número de diputadas que corresponde a 4,75\%, entre un ciclo legislativo y el anterior. 
Tabla 5. Variación del porcentaje de mujeres, según escenario electoral

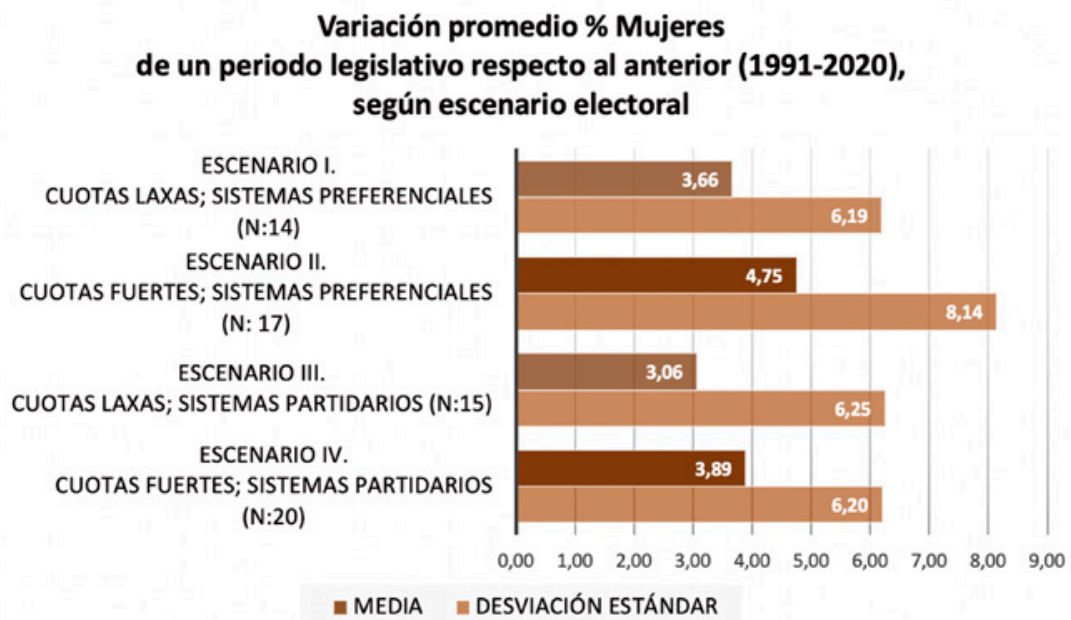

Elaboración propia según leyes electorales del país, bases de datos de Quotaproyect, IDEA International, Inter-parliamentary Union y Aceproject, Casas-Zamora et al., 2016, Archenti y Tula, 2013.

De acuerdo con la tabla, el análisis pone en evidencia que un mayor protagonismo de la ciudadanía en la selección de sus representantes no comporta un retroceso en la representación femenina cuando se acompaña de leyes de cuotas bien definidas. Sin embargo, también los sistemas electorales de tipo partidario resultan dar buenos resultados en término de representación descriptiva cuando están acompañados por cuotas fuertes. Asimismo, se puede observar que el impacto de las leyes de cuotas en la variación de mujeres elegidas en los dos escenarios al extremo del espectro (cuotas de género laxas y orientación preferencial; cuotas de género fuertes y orientación partidaria) resulta parecido, aunque se registra en promedio un indicio de una mayor variación positiva en los sistemas no preferenciales con cuotas de género bien dibujadas.

\section{6.- Conclusiones}

El presente estudio ha examinado el impacto de las leyes de cuotas de género en la representación descriptiva de las mujeres latinoamericanas en política. A partir de una extensa literatura disponible, se ha puesto de relieve que la presencia de mujeres legisladoras tiene una importante relevancia a nivel sustantivo, como condición fundamental para alcanzar un mayor nivel de calidad democrática y una convivencia libre de discriminación por género. En el análisis se ha podido incluir trece países latinoamericanos y sesenta y seis periodos legislativos, a lo largo de 
casi treinta años, desde 1991 hasta 2020, con el objetivo de explorar la vinculación entre los niveles de acceso de las mujeres al legislativo y las reglas electorales. Los factores analizados han sido los elementos del corpus normativo de leyes de cuotas de género vigentes a nivel de elecciones nacionales, y la estructura de la papeleta de votación en términos de orientación preferencial o partidaria del sistema electoral.

En definitiva, este trabajo de investigación transnacional aspira a ser un aporte al debate sobre el papel de las cuotas de género en favorecer el acceso de las mujeres a los espacios de poder y el condicionamiento del voto preferencial sobre la eficiencia de esta medida de acción afirmativa. En el análisis se ha podido observar que el contenido de las leyes de cuotas es un aspecto particularmente relevante a la hora de examinar la evolución de la representación femenina en el tiempo. En particular, las leyes de cuotas fuertes que incorporan un porcentaje de presentación mínima entre el 40 y el 50\%, un mandato de posición y sanciones fuertes son las que inciden mayormente sobre la variación de cargos electivos ganados por mujeres, independientemente del tipo de lista electoral presente. Además, cuando se enfoca el análisis en la estructura de la papeleta de votación, se observa que los sistemas electorales con voto preferencial no perjudican el avance de las mujeres latinoamericanas en política. Aunque la presente investigación haya generado valiosas reflexiones sobre la relación entre las reglas electorales y el impacto de las cuotas de género, se recomienda estudios adicionales que examinen la incidencia de un número más elevado de variables sobre el avance de la participación de las mujeres en política.

\section{Referencias Bibliográficas}

ALCÁNTARA SÁEZ, Manuel et al. (2008) Reformas económicas y consolidación democrática. Historia contemporánea de América Latina, Volumen VI, 1980-2006. ÍCONOS, 30.

ALCÁNTARA SÁEZ, Manuel et al. (2013) Procesos politicos y electorales en América Latina (2010- 2013), Buenos Aires: Eudeba.

ARCHENTI, Nélida (2009) ¿Qué piensan los líderes de opinión de América Latina sobre la paridad y la participación de las mujeres en la política?, Santiago de Chile: CEPAL.

ARCHENTI, Nélida (2011) La paridad política en América Latina y el Caribe: Percepciones y opiniones de los líderes de la región, Mujer y Desarrollo, 108.

ARCHENTI, Nélida y María Inés TULA (2007) «Cuotas de género y tipo de lista en América Latina», Opinião Pública, 13(1), pp.185-218.

ARCHENTI, Nélida y María Inés TULA (2013) «¿Las mujeres al poder? Cuotas y paridad de género en América Latina», Instituto de Iberoamérica, Universidad de Salamanca, Seminario de Investigación \#9.

Autor 1 (2016) Understanding differences across EU member states when assessing the impact of gender quotas (Tesis de fin de Grado no publicada). University 1. 
BACCHI, Carol Lee (1996) The politics of affirmative action: 'Women', equality and category politics, London: Sage Publications.

BALLIVIÁN, Salvador Romero (2016) «Orientaciones, dilemas y desafíos de las reformas electorales en América Latina en el inicio del siglo XXI», en K. Casas -Zamora et al. (Eds.), Reformas políticas en América Latina: tendencias y casos (pp.93-133), Washington DC: SG/OEA.

BAREIRO, Line et al. (2004) Sistemas electorales y representación femenina en América Latina, United Nations Publications.

CASAS-ZAMORA, Kevin et al. (2016) Reformas políticas en América Latina: tendencias y casos, Washington DC: SG/OEA.

CAUL, Miki (2001) «Political parties and the adoption of candidate gender quotas: A cross-national analysis», Journal of Politics, 63 (4), pp. 1214-1229.

CELIS, Karen et al. (2008) «Rethinking women's substantive representation», Representation, 44 (2), pp. 99-110.

CEPAL, NNUU (2016) Autonomía de las mujeres e igualdad en la agenda de desarrollo sostenible, Santiago de Chile: NNUU.

CEPAL, NNUU (2017a) Agenda 2030 y los Objetivos de Desarrollo Sostenible: una oportunidad para América Latina y el Caribe, Santiago de Chile: NNUU.

CEPAL, NNUU (2017c) Planes de igualdad de género en América Latina y el Caribe Mapas de ruta para el desarrollo, Santiago: NNUU.

CEPAL, NNUU. (2017b, 27 de mayo) «Ministras y altas autoridades de la Mujer revisan en la CEPAL puesta en marcha de la Estrategia de Montevideo para la igualdad de género». Comunicados de prensa. Disponible en: http:/ / www.cepal. org/es/comunicados/ministras- altas-autoridades-la-mujer-revisan-la-cepalpuesta-marcha-la-estrategia (Fecha de consulta: 13 de julio 2019).

CHILDS, Sarah y Lena Mona KROOK (2008) «Critical mass theory and women's political representation», Political studies, 56(3), pp. 725-736.

DAHLERUP, Drude y Lenita FREIDENVALL (2005) «Quotas as a 'fast track'to equal representation for women: Why Scandinavia is no longer the model», International Feminist Journal of Politics, 7(1), pp. 26-48.

DAHLERUP, Drude y Lenita FREIDENVALL (2008) Electoral gender Quota Systems and Their Implementation in Europe: Issued in English, French, German, Italian and Spanish.

DAHLERUP, Drude y Lenita FREIDENVALL (2010) «Judging gender quotas: predictions and results»,

Policy E Politics, 38(3), pp. 407-425.

DAHLERUP, Drude y Lenita FREIDENVALL (2011) «Electoral gender quota systems and their implementation in Europe», Policy Department C: Citizens' Rights And Constitutional Affairs, 1-170. Disponible en: http://www.europarl.europa. eu/studies (Fecha de consulta: 1 de julio 2019).

DALTON, Russel J., FARRELL, David M. y Ian MCALLISTER (2011) Political parties and democratic linkage: How parties organize democracy, Oxford: Oxford University Press.

de Quito, Consenso (2007) Décima Conferencia Regional sobre la Mujer de América Latina y el Caribe, Quito, Ecuador.

Decreto $N^{\circ}$ 1246. Argentina, 2000. Disponible en: https://reformaspoliticas.org/ 
normativa/ temas/leyes-de-cuotas/ (Fecha de consulta: 3 de junio 2020).

DIELEMAN, Ruben y Frida ANDERSSON (2016) «Measuring women's political empowerment and investigating the role of women's civil liberties in democratization», V-dem Policy Brief, 4, pp. 1-3.

DISCH, Lisa (2011) «Toward a mobilization conception of democratic representation», American Political Science Review, 105 (1), pp. 100-114.

FARRELL, David M. y Ian MCALLISTER (2006) «Voter satisfaction and electoral systems: Does preferential voting in candidate-centred systems make a difference?», European Journal of Political Research, 45(5), pp. 723-749.

FARRELL, David M. y Roger SCULLY (2007) Representing Europe's citizens?: electoral institutions and the failure of parliamentary representation, Oxford University Press.

FRANCESCHET, Susan y Jennifer PISCOPO (2008) «Gender quotas and women's substantive representation: Lessons from Argentina», Politics \& Gender, 4(3), pp. 393-425.

FRÉCHETTE, Guilliaume. R., MANIQUET, Francois y Massimo MORELLI (2008) «Incumbents' interests and gender quotas», American Journal of Political Science, 52(4), pp. 891-909.

FREIDENBERG, Flavia et al. (Eds.) (2018) Mujeres en la Política: experiencias nacionales y subnacionales en América Latina, Instituto Electoral de la Ciudad de México.

FREIDENBERG, Flavia y Gabriela DEL VALLE PÉREZ (2017) Cuando hacer política te cuesta la vida. Estrategias contra la Violencia Política hacia las Mujeres, Instituto de Investigaciones Jurídicas, Seri Doctrina Jurídica: México.

FREIDENBERG, Flavia y Tomas DOŠEK (2016) «Las reformas electorales en América Latina (1978- 2015)», en K. Casas-Zamora et al. (Eds.), Reformas políticas en América Latina: tendencias y casos (pp. 25-92). Washington DC: SG/OEA.

HTUN, Mala. N. y Mark P. JONES (2002) «Engendering the right to participate in decision-making: electoral quotas and women's leadership in Latin America», en Gender and the politics of rights and democracy in Latin America (pp. 32-56). London: Palgrave Macmillan UK.

IDEA International (2020) Base de Dato de Cuotas. Disponible en: http://www. idea.int/data- tools/data/gender-quotas (Fecha de consulta: 7 de marzo 2020).

IDEA, Stockholm University y Inter-Parliamentary Union (2020a) Quota Project: Research database. Disponible en: http://www.quotaproject.org/uid/search. cfm (Fecha de consulta: 20 de marzo 2020).

IDEA, Stockholm University y Inter-Parliamentary Union (2020b) Quota Project: Country Overview. Disponible en: http://www.quotaproject.org/country. cfm?SortOrder=Country (Fecha de consulta: 7 de marzo 2020).

IDEA, Stockholm University y Inter-Parliamentary Union (2020c) Quota Project: Argentina. Disponible en: http://www.quotaproject.org/country/ argentina\#party (Fecha de consulta: 12 de marzo 2020).

IDEA, Stockholm University y Inter-Parliamentary Union (2020d) Quota Project: Brazil. Disponible en: http://www.quotaproject.org/country/brazil (Fecha de consulta: 12 de marzo 2020).

INGLEHART, Ronald, NORRIS, Pipa y Inglehart RONALD (2003) Rising tide: Gen- 
der equality and cultural change around the world, Cambridge: Cambridge University Press.

Inter-Parliamentary Union (2020a) PARLINE Database on National Parliaments. Disponible en: http://www.ipu.org/parline-e/parlinesearch.asp (Fecha de consulta: 12 de abril 2020).

Inter-Parliamentary Union (2020b) EL SALVADOR: Asamblea legislativa. Disponible en: http:/ / www.ipu.org/parline-e/reports /2099_E.htm (Fecha de consulta: 12 de marzo 2020).

JONES, Mark P. (2009) «Gender quotas, electoral laws, and the election of women: Evidence from the Latin American vanguard», Comparative Political Studies, 42(1), pp. 56-81.

JONES, Mark P. y Patricio NAVIA (1999) «Assessing the effectiveness of gender quotas in open-list proportional representation electoral systems», Social Science Quarterly, pp. 341-355.

JONES, Mark P., ALLES, Santiago y Carolina TCHINTIAN (2012) «Cuotas de género, leyes electorales y elección de legisladoras en América Latina», Revista de ciencia política (Santiago), 32(2), pp. 331-357.

KARVONEN, Lauri (2004) «Preferential voting: Incidence and effects», International Political Science Review, 25(2), pp. 203-226.

KITTILSON, Miki Caul (2006) Challenging parties, changing parliaments: Women and elected office in contemporary Western Europe, Ohio State University Press.

KROOK, Mona Lena (2006) «Reforming representation: The diffusion of candidate gender quotas worldwide», Politics E Gender, 2(3), pp. 303-327.

KROOK, Mona Lena (2007) «Quotas for women in elected politics: Measures to increase women's political representation worldwide», Towards Achieving at Least, 30, pp. 3-7.

LARSERUD, Stina y Rita TAPHORN (2007) Diseño para lograr la igualdad: combinaciones más idóneas, medianamente idóneas y no favorables de los sistemas electorales y cuotas de género, International Institute for Democracy and Electoral Assistance (International IDEA).

Ley N²4.012. Argentina, 1991. Disponible en: https://reformaspoliticas.org/normativa/temas/leyes- de-cuotas/ (Fecha de consulta: 3 de junio 2020).

LLANOS, Beatriz (2019) «Sulcando Olas y Contra-olas: Una mirada paritaria a los derechos políticos de las mujeres en América Latina», IDEA.

MANSBRIDGE, Jane (1999) «Should blacks represent blacks and women represent women? A contingent «yes»», The Journal of politics, 61(3), pp. 628-657.

MANSBRIDGE, Jane (2005) «Quota problems: Combating the dangers of essentialism», Politics $\mathcal{E}$ Gender, 1(4), pp. 622-638.

MATLAND, Richard E. (1993) «Institutional variables affecting female representation in national legislatures: The case of Norway», The Journal of Politics, 55(3), pp. 737-755.

MATLAND, Richard E. y Donley T. STUDLAR (1996) «The contagion of women candidates in single- member district and proportional representation electoral systems: Canada and Norway», The journal of politics, 58(3), pp. 707-733.

MCELROY, Gail y Michael MARSH (2010) «Candidate gender and voter choice: 
Analysis from a multimember preferential voting system», Political Research Quarterly, 63(4), pp. 822-833.

MIRANDA CUESTAS, Guillermo (2016) «La reforma electoral en El Salvador de 1992 a 2015: más derechos sin mejores instituciones», en K. Casas-Zamora et al. (Eds.), Reformas politicas en América Latina: tendencias y casos (pp. 227-245). Washington DC: SG/OEA.

MURRAY, Rainbow (2010) Parties, gender quotas and candidate selection in France, Basingstoke: Springer.

PACHANO, Simón (2004) El proceso electoral de Ecuador, Quito, Ecuador: FLACSO.

ParlGov (2020) Dataset. Disponible en: http://www.parlgov.org/static/static-2014/stable/data.html (Fecha de consulta: 25 de marzo 2020).

PAXTON, Pamela y Sheri KUNOVICH (2003) «Women's political representation: The importance of ideology», Social Forces, 82(1), pp. 87-113.

PAXTON, Pamela, HUGHES, Melanie M. y Matthew A. PAINTER (2010) «Growth in women's political representation: A longitudinal exploration of democracy, electoral system and gender quotas», European Journal of Political Research, 49(1), pp. 25-52.

PESCHARD, Jacqueline (2003) «The quota system in Latin America: General overview», en IDEA workshop on the Implementation of Quotas: Latin American Experiences, Lima (pp. 20-29), Suecia: Trydells Tryckeri AB.

PHILLIPS, Anne (1995) The politics of presence, Oxford: Clarendon Press.

PHILLIPS, Anne (1998) «Democracy and representation: Or, why should it matter who our representatives are? », Feminism and politics, pp. 224-240.

PITKIN, Hanna (1964) «Hobbes' Concept of Representation», American Political Science Review, 58(2), pp. 328-340.

PITKIN, Hanna (1967) The concept of representation, California: Univ of California Press.

RAI, Shirin M. (2013) The gender politics of development: essays in hope and despair, London: Zed Books Ltd.

SCHMIDT, Gregory D. (2003) «Unanticipated Successes: Lessons from Peru's Experiences with Gender Quotas in Majoritarian Closed List and Open List PR Systems», IDEA, lc, pp. 120- 133.

SCHWINDT-BAYER, Leslie A. (2009) «Making quotas work: The effect of gender quota laws on the election of women», Legislative studies quarterly, 34(1), pp. 5-28.

SCHWINDT-BAYER, Leslie A. (2010) Political power and women's representation in Latin America, Oxford: Oxford University Press.

SCHWINDT-BAYER, Leslie A. y William Mishler (2005) «An integrated model of women's representation», Journal of Politics, 67(2), pp. 407-428.

SOLDEVILLA, Fernando Tuesta (2005) «Sistemas electorales en América Latina», Revista IIDH,

Vol.42, pp. 211-225.

SQUIRES, Judith (2007) The new politics of gender equality, New York: Palgrave Macmillan. SUNDSTRÖM Asker et al. (2017) «Women's Political Empowerment: A New Global Index, 1900-2012», World Development, 94, pp. 321-335.

TRIPP, Aili Mari y Alice KANG (2008) «The global impact of quotas: On the fast 
track to increased female legislative representation», Comparative Political Studies, 41(3), pp. 338-361.

TULA, María Inés (2016) «Mujeres y política: un panorama sobre la adopción de las cuotas de género y sus efectos en América Latina y Colombia», en K. CasasZamora et al. (Eds.), Reformas políticas en América Latina: tendencias y casos (pp. 25-92). Washington DC: SG/OEA.

V-Dem Institute (2020) Online Analysis Tool. Disponible en: https: / /www.v- dem. net/en/analysis/analysis/ (Fecha de consulta: 12 de marzo 2020).

WÄNGNERUD, Lena (2009) «Women in parliaments: Descriptive and substantive representation», Annual Review of Political Science, 12, 51-69.

World Value Survey (2017b) Changing Attitudes towards Gender Equality: Update from the World Values Survey. Documentation. Disponible en: https://paa.confex. com/paa/2017/mediafile/ExtendedAbstract/Paper15576/rsPAA\%202017

_gender\%20wvs_long\%20abstract.pdf (Fecha de consulta: 06 de marzo 2017).

World Value Survey (2020a) Online Data Analysis. Disponible: http:/ / www.worldvaluessurvey.org/WVSOnline.jsp (Fecha de consulta: 12 de marzo 2020). YOUNG, Iris Marion (2002) Inclusion and democracy, Oxford: Oxford University Press on Demand.

ZAPATA, Daniela (2007) Transversalizando la perspectiva de género en los objetivos de desarrollo del Milenio, Santiago de Chile: CEPAL.

Recibido el 30 de junio de 2020 Aceptado el 13 de marzo de 2021 BIBLID [1132-8231 (2021): 17-39] 